\title{
Estimating the burden of cervical disease among HIV-infected women accessing screening services in South Africa: A model-based analysis
}

\author{
C J Chibwesha, ${ }^{1,2}$ MD, MSc, FACOG; B Goeieman, ${ }^{1}$ MB BCh; S Levin, ${ }^{1}$ FRCOG; M Mulongo, ${ }^{1}$ MB BCh; M Faesen, ${ }^{1}$ FCOG (SA); \\ A Swarts, ${ }^{1,3}$ MSc; S Ramotshela ${ }^{1,3}$ S Williams; ${ }^{1}$ N Rakhombe; ${ }^{1}$ S Bruce; ${ }^{1}$ P Michelow, ${ }^{4,5}$ MB BCh, MSc; C Firnhaber, ${ }^{1,3}$ MD, MS \\ ${ }^{1}$ Right to Care, Helen Joseph Hospital, Johannesburg, South Africa \\ ${ }^{2}$ Division of Global Women's Health, Department of Obstetrics and Gynecology, School of Medicine, University of North Carolina at Chapel Hill, \\ NC, USA \\ ${ }^{3}$ Clinical HIV Research Unit, Department of Internal Medicine, School of Clinical Medicine, Faculty of Health Sciences, University of the \\ Witwatersrand and Helen Joseph Hospital, Johannesburg, South Africa \\ ${ }^{4}$ Department of Anatomical Pathology, School of Pathology, Faculty of Health Sciences, University of the Witwatersrand, Johannesburg, South Africa \\ ${ }^{5}$ National Health Laboratory Service, Johannesburg, South Africa
}

Corresponding author: C J Chibwesha (carla_chibwesha@med.unc.edu)

\begin{abstract}
Background. Cervical cancer remains the second most common cancer among women worldwide, with much of the global burden occurring in low- and middle-income countries. HIV-infected women are at increased risk of human papillomavirus infection, preinvasive cervical disease and invasive cervical cancer (ICC). Funded through the United States President's Emergency Plan for AIDS Relief (PEPFAR) and working in collaboration with the South African (SA) Department of Health, our team supports cervical screening integrated within public sector HIV clinics in SA.

Objectives. To estimate the burden of cervical disease among HIV-infected women accessing screening services supported through our programme. Methods. We constructed conditional probability models to estimate the burden of grade 1 and grades $2 / 3$ cervical intraepithelial lesions (CIN1 and CIN2/3) and ICC among two cohorts: one consisting of 3190 HIV-infected women for whom only cytology results were available for analysis, and another consisting of $75358 \mathrm{HIV}$-infected women for whom neither cytology nor histology results were available. Parameter estimates for the models were derived from routinely collected programmatic data and published clinical trials.

Results. Between January 2009 and November 2015, 75358 HIV-infected women underwent Pap smear screening in public sector clinics supported by our cervical cancer prevention programme. Based on modelling analysis, we estimate that 46123 cases of CIN1 (range 45500 49 608), 13598 cases of CIN2/3 (range 12749 - 14 828), and 104 cases of ICC (range 61 - 186) occurred in this population.

Conclusions. Our findings highlight the magnitude of cervical disease among HIV-infected women in SA.
\end{abstract}

S Afr Med J 2018;108(3):235-239. DOI:10.7196/SAMJ.2018.v108i3.12627

Despite the availability of cost-effective prevention strategies, cervical cancer remains among the most common cancers worldwide. ${ }^{[1]}$ In sub-Saharan Africa, where the vast majority of disease occurs, cervical cancer is the leading cause of cancer death among women. ${ }^{[2]}$ The relative dearth of organised prevention programmes and a high burden of HIV contribute to the region's high cervical cancer rate. Thirteen million women are currently infected with HIV in subSaharan Africa. ${ }^{[3]}$ These women are at significantly increased risk of developing a persistent high-risk human papillomavirus infection (the necessary cause of most cervical cancers), preinvasive cervical disease and invasive cervical cancer (ICC). ${ }^{[4]}$ Cervical disease also appears to be harder to treat in HIV-infected women, with lower cure rates achieved in this population. ${ }^{[5,6]}$

South Africa (SA) is home to the largest number of HIV-infected individuals in the world, ${ }^{[3]}$ with nearly $60 \%$ of incident HIV infections occurring in women. ${ }^{[7]}$ The country's age-standardised incidence of cervical cancer is 32 per $100000,{ }^{[8]}$ which is one of the highest rates in the world. 2010 Southern African HIV Clinicians Society guidelines recommend cervical screening with cytology (Pap smears) beginning at the time of HIV diagnosis, and at 3-year intervals thereafter for women who screen negative. If implemented fully and correctly, this screening strategy is expected to reduce women's lifetime risk of developing cervical cancer substantially ${ }^{[9]}$ However, as in many other low- and middle-income countries (LMICs), achieving meaningful cervical screening coverage has been elusive. In fact, effective population coverage for cervical screening is thought to be $<20 \%$ in SA. ${ }^{[10]}$ There are several reasons for this. First, cancer prevention has not received sufficient public policy attention or funding. Second, the cost and complex nature of the Pap smear have made cytology-based screening difficult to scale up, particularly to rural and remote communities. Third, public awareness of and advocacy for cervical cancer prevention remain relatively low, resulting in weak demand for services. ${ }^{[11]}$

\section{Objectives}

To estimate the burden of cervical disease among HIV-infected women accessing cervical screening services supported through the Right to Care Cervical Cancer Prevention Programme, a large donorfunded public sector programme.

\section{Methods \\ Design}

To estimate the programme-wide burden of cervical disease, we developed conditional probability models using parameter estimates obtained from both a secondary analysis of routinely collected data 
(described below) and previously published research. ${ }^{[12]}$ We chose to model disease burden because patient-level cervical histology data are not available from all clinical sites supported by our programme.

\section{Service delivery programme platform}

Established in 2009, Right to Care's Cervical Cancer Prevention Programme has provided services to $>80000$ women using either cytology $(n=75358)$ or visual inspection with acetic acid (VIA) ( $n=5$ 532) in Eastern Cape, Free State, Gauteng, Mpumalanga and Western Cape provinces. Pap smear screening is typically nurse led, with selected rural sites instead performing VIA. Mobile women's health units are employed in under-served areas of the Free State, Gauteng, Mpumalanga and Western Cape. These mobile units also provide a variety of other reproductive health services (e.g. clinical breast examinations, screening for sexually transmitted infections, and contraception). Women with abnormal Pap smear or VIA results receive follow-up evaluation (i.e. colposcopy) and any clinically indicated treatment at either the screening clinic or a referral centre. Our team also trains medical officers to perform cervical colposcopy and loop electrosurgical excision procedures (LEEPs). Approximately 23000 colposcopies and LEEPs have been performed since the inception of the programme.

Primary funding for service delivery is provided through the US President's Emergency Plan for AIDS Relief (PEPFAR), and service implementation occurs in partnership with the SA Department of Health (DoH). Mobile services are supported through a unique public-private partnership with the SA insurance company First for Women. This enables our cervical cancer prevention services to be provided free of user fees.

\section{Secondary analysis of programmatic data}

Of the 25 public sector clinical sites supported by our programme, six were selected for enhanced monitoring and evaluation (M\&E) activities. These sites, representing urban, periurban and rural areas of Gauteng and Mpumalanga, collect and report individual-level sociodemographic, HIV treatment and cervical cytology data for all women accessing Pap smear services. (Patient-level data are not available from the other 19 sites.)

In this report, we present the available data from these six enhanced M\&E sites. Descriptive analysis included frequencies, measures of central tendency and measures of variability, which were generated for sociodemographic, clinical and laboratory variables. Considering only the index Pap smear, we report cytology results in accordance with the 2001 Bethesda System as no intraepithelial lesion or malignancy (NILM), atypical cells of undetermined significance (ASCUS), atypical glandular cells of undermined significance (AGUS), low-grade squamous intraepithelial lesion (LSIL), highgrade SIL (HSIL), atypical squamous cells, cannot rule out a highgrade lesion (ASC-H), or suspicious for ICC. ${ }^{[13]}$ We calculated the point estimates and corresponding $95 \%$ confidence intervals (CIs) for each of these cytological categories. We also examined the association between abnormal cytology (ASCUS or worse (ASCUS+), LSIL or worse (LSIL+), and HSIL or worse (HSIL+)) and both CD4+ cell count and receipt of antiretroviral therapy (ART). HIV-1 plasma viral load data were not available.

All data were cleaned using MS Excel, version 15.36 (Microsoft, USA) and then analysed using Stata version 12.1 (StataCorp, USA).

\section{Conditional probability modelling to estimate the burden of cervical disease}

First, we modelled the burden of cervical disease (i.e. abnormal histology) among the subset of women from our programme's enhanced M\&E sites ( $N=3$ 160). We used the frequencies of NILM, ASCUS, AGUS, LSIL, HSIL and cytology suspicious for ICC obtained from our programmatic database. We then estimated the expected number of NILM, cervical intraepithelial neoplasia grade 1 (CIN1), CIN grade 2 (CIN2), CIN grade 3 (CIN3), and ICC cases using the probability of observing each histological diagnosis conditional on each of the above cytological diagnoses. The histological (i.e. disease) probability estimates were obtained from a previous study conducted among HIV-infected women in Johannesburg. ${ }^{[12]}$ Specifically, the probabilities of NILM, CIN1, CIN2, CIN3 and ICC were assumed to be $0.465,0.482,0.053,0$ and 0 , respectively, among women with a NILM Pap smear; 0.103, 0.828, 0.069, 0 and 0, respectively, among women with an ASCUS Pap smear; 0.057, 0.803, 0.128, 0.012 and 0, respectively, among women with an LSIL Pap smear; 0.011, 0.313, $0.408,0.261$ and 0.006 , respectively, among women with an HSIL Pap smear; $0.103,0.828,0.069,0$ and 0 , respectively, among women with an AGUS Pap smear; and 0, 0, 0, 0.500 and 0.500, respectively, among women with a Pap smear suspicious for ICC.

Second, we modelled the programme-wide burden of cervical disease, estimating the frequencies of NILM, CIN1, CIN2, CIN3 and ICC expected among all HIV-infected women accessing services through our programme $(N=75358)$ but for whom individuallevel information was not available. We used the probability of NILM, ASCUS, LSIL, ASC-H, HSIL and cytology suspicious for ICC observed in our programmatic database to model the expected frequency of each Pap smear finding. We then calculated the expected number of NILM, CIN1, CIN2, CIN3 and ICC cases using the parameter estimates from our published work, ${ }^{[12]}$ as described above. Sensitivity analyses were conducted to provide low and high estimates of disease burden using the lower and upper bounds of the 95\% CIs for NILM, ASCUS, LSIL, ASC-H, HSIL and cytology suspicious for ICC derived from programmatic data. Once again, we used a conditional probability modelling approach to determine low and high estimates of the number of cases of NILM, CIN1, CIN2, CIN3 and ICC.

All conditional probability models were created using MS Excel, version 15.36 (Microsoft, USA).

\section{Ethical considerations}

Ethical approval for secondary analysis of routine clinical data from the six clinical sites with enhanced M\&E activities was obtained from the Human Research Ethics Committee, University of the Witwatersrand, Johannesburg (ref. no. M10956). All the women accessing services at these sites provided written informed consent for data collection.

\section{Results}

Between January 2009 and November 2015, 3190 HIV-infected women accessed cervical screening services at a site from which individual-level data were available. Thirty women were excluded from the analysis because their Pap smear result was missing. The median age of the remaining 3160 women was 37 years (interquartile range (IQR) 16 - 73). Most women had completed some secondary education (64.5\%), and nearly half $(47.8 \%)$ were employed. As expected, most women (77.9\%) were receiving combination ART (cART), with a median CD4+ cell count of 348 cells/ $\mu \mathrm{L}$ (IQR 208 517) (Table 1).

Cytology results for the subset of $3160 \mathrm{HIV}$-infected women are detailed in Table 1. Notably, 2012 of these women (63.7\%) had an ASCUS+ cytology result and 1610 (50.9\%) had an LSIL+ cytology result. Additionally, women with $\mathrm{CD} 4+$ cell counts $\leq 350$ cells $/ \mu \mathrm{L}$ were more likely to have an abnormal Pap smear than those with higher counts $(p<0.001)$ (Table 2). However, receipt of cART did 
not appear to decrease the risk of an abnormal Pap smear at the statistically significant level (data not shown).

In Tables 3 and 4, we provide estimates of the burden of cervical disease in both our analysis cohort and the programme at large. In the analysis cohort, consisting of 3160 women, we estimate that there would have been 1937 cases of CIN1 (61.3\%) and 578 cases of CIN2+ (18.3\%). Similarly, among $75358 \mathrm{HIV}$-infected women we estimate that 27355 would have had NILM, 9570 ASCUS, 527 AGUS, 26074

\begin{tabular}{|c|c|}
\hline & Total, $n(\%)$ \\
\hline Age (years), median (IQR) ( $N=3160)$ & $37(16-73)$ \\
\hline \multicolumn{2}{|l|}{ Education, $n(\%)(N=3$ 160) } \\
\hline No education & $221(7.0)$ \\
\hline Primary education & $765(24.0)$ \\
\hline Secondary education & $2037(64.5)$ \\
\hline Tertiary education & $146(4.6)$ \\
\hline \multicolumn{2}{|l|}{ Employment, $n(\%)(N=3160)$} \\
\hline Yes & $1512(47.8)$ \\
\hline \multicolumn{2}{|l|}{ Cigarette smoking, $n(\%)(N=3$ 160) } \\
\hline Yes & $80(2.5)$ \\
\hline Age at sexual debut, median (IQR) ( $N=3156)$ & $18(17-24)$ \\
\hline Parity, median (IQR) $(N=3160)$ & $2(1-3)$ \\
\hline \multicolumn{2}{|l|}{ Hormonal contraception, $n(\%)(N=3160)$} \\
\hline None & $2448(77.5)$ \\
\hline Oral contraceptives & $95(3.0)$ \\
\hline Injectables & $600(19.0)$ \\
\hline Other & $17(0.5)$ \\
\hline \multicolumn{2}{|l|}{ On antiretroviral therapy, $n(\%)(N=3160)$} \\
\hline Yes & $2460(77.9)$ \\
\hline \multicolumn{2}{|l|}{$(N=3137)$} \\
\hline \multicolumn{2}{|l|}{ Cytology, $n(\%)(N=3160)$} \\
\hline NILM & $1148(36.3)$ \\
\hline ASCUS & $402(12.7)$ \\
\hline AGUS & $23(0.7)$ \\
\hline LSIL & $1094(34.6)$ \\
\hline HSIL & 489 (15.5) \\
\hline Suspicious for ICC & $4(0.1)$ \\
\hline \multicolumn{2}{|c|}{ 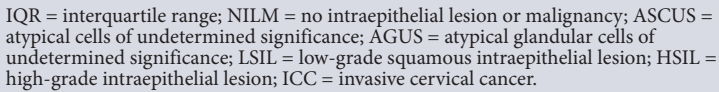 } \\
\hline
\end{tabular}

LSIL and 11605 HSIL Pap smears. We also estimate that 75 women would have had Pap smears suspicious for ICC. Conditional on these cytology results, we estimate 46123 cases of CIN1 (range 45500 - 49 608) and 13702 cases of CIN2+ (range 12810 - 15 014), constituting an overwhelmingly high burden of disease.

\section{Discussion}

Sub-Saharan Africa is the global epicentre of the HIV epidemic: 70\% of the 36.7 million people currently living with HIV are African, and 14.2 million are African women. ${ }^{[3]}$ Although the introduction of cART has led to reductions in the incidence of cancers such as Kaposi's sarcoma and non-Hodgkin's lymphoma among HIVinfected individuals, ${ }^{[14]}$ the role of ART in reducing the incidence of ICC and its precursors remains unclear. ${ }^{[15]}$ As increasing numbers of HIV-infected women initiate ART in places such as SA, we anticipate that longer life expectancy for these women will be coupled with a rising burden of chronic diseases, including ICC. ${ }^{[16,17]}$

Our model-based analysis indicates that nearly one in five HIVinfected women in our programme will have been diagnosed with high-grade preinvasive cervical cancer requiring treatment with LEEP and close clinical follow-up to ensure that disease recurrence or progression is appropriately managed. Additionally, $61.3 \%$ of women were estimated to have CIN1, also requiring close clinical follow-up. Most strikingly, 104 cases of ICC were projected in this population, corresponding to a crude rate of 138 cases per 100000 women (range $81-247$ ). This burden of ICC in HIV-infected women appears to be nearly five times the national age-standardised incidence of 32 per $100000,{ }^{[8]}$ and is consistent with previous reports suggesting a two- to seven-fold increase in the risk of ICC among HIV-infected women. ${ }^{[18-20]}$

Our findings underscore how deeply neglected cancer prevention has been in settings such as SA, and raise concerns that high rates of ICC in HIV-infected women threaten to undo - or even reverse - gains in life expectancy achieved through HIV treatment programmes. Our results also suggest that continued advocacy and health service expansion are needed to improve cervical screening coverage. Below, we describe our service delivery model as an example that may be considered in similar settings.

Our cervical cancer prevention services are integrated into public sector HIV clinics to ensure that HIV-infected women can have full and timely access to cervical screening. Early in programme planning, we also recognised the far-reaching impact that constrained human resources can have on service delivery. To address the shortage of trained personnel, our team provides in-service training to $\mathrm{DoH}$ nurses and general medical officers. In the 6 years since our

Table 2. Risk of abnormal cytology by CD4+ cell count

\begin{tabular}{|c|c|c|c|}
\hline & Total, $N$ & $\begin{array}{l}\text { CD4+ cell count } \\
\leq 350 \text { cells } / \mu \mathrm{L}, n(\%)\end{array}$ & $\begin{array}{l}\text { CD4+ cell count } \\
>350 \text { cells } / \mu \mathrm{L}, n(\%)\end{array}$ \\
\hline \multicolumn{4}{|c|}{ Using an ASCUS+ cutoff } \\
\hline NILM & 1140 & $428(37.5)$ & $712(62.5)$ \\
\hline ASCUS+ & 1997 & $1161(58.1)$ & $836(41.9)^{*}$ \\
\hline \multicolumn{4}{|c|}{ Using an LSIL+ cutoff } \\
\hline$<\mathrm{LSIL}$ & 1540 & $624(40.5)$ & $916(59.5)$ \\
\hline LSIL+ & 1597 & $965(60.4)$ & $632(39.6)^{*}$ \\
\hline \multicolumn{4}{|c|}{ Using an HSIL+ cutoff } \\
\hline$<$ HSIL & 2623 & $1260(48.0)$ & $1363(52.0)$ \\
\hline HSIL+ & 514 & $329(64.0)$ & $185(36.0)^{*}$ \\
\hline
\end{tabular}


Table 3. Estimated burden of histological disease among HIV-infected women for whom individual-level cytology data were available $(N=3160)$

\begin{tabular}{|c|c|c|c|c|c|c|}
\hline \multirow[b]{2}{*}{ Cytology } & \multirow[b]{2}{*}{$N$} & \multicolumn{5}{|c|}{ Estimated disease burden, $n$} \\
\hline & & NILM $^{*}$ & CIN1* $^{*}$ & CIN2* $^{*}$ & $\mathrm{CIN}^{*}$ & $\mathrm{ICC}^{\dagger}$ \\
\hline NILM & 1148 & 534 & 554 & 60 & 0 & 0 \\
\hline ASCUS & 402 & 42 & 333 & 28 & 0 & 0 \\
\hline AGUS & 1094 & 62 & 878 & 140 & 13 & 0 \\
\hline LSIL & 489 & 6 & 153 & 200 & 128 & 3 \\
\hline HSIL & 23 & 2 & 19 & 2 & 0 & 0 \\
\hline Suspicious for ICC & 4 & 0 & 0 & 0 & 2 & 2 \\
\hline Total & 3160 & 646 & 1937 & 430 & 143 & 5 \\
\hline
\end{tabular}

NILM = no intraepithelial lesion or malignancy; ASCUS = atypical cells of undetermined significance; AGUS = atypical glandular cells of undetermined significance; LSIL = low-grade squamous intraepithelial lesion; HSIL = high-grade intraepithelial lesion; CIN = cervical intraepithelial neoplasia (grades 1, 2 and 3); ICC = invasive cervical cancer

${ }^{+}$Owing to the lack of robust data for HIV-infected women in our setting, probabilities of CIN3 and ICC are estimated as 0.500 for cytology results suspicious for ICC.

Table 4. Estimated burden of histological disease among HIV-infected women accessing care through Right to Care's Cervical Cancer Prevention Programme

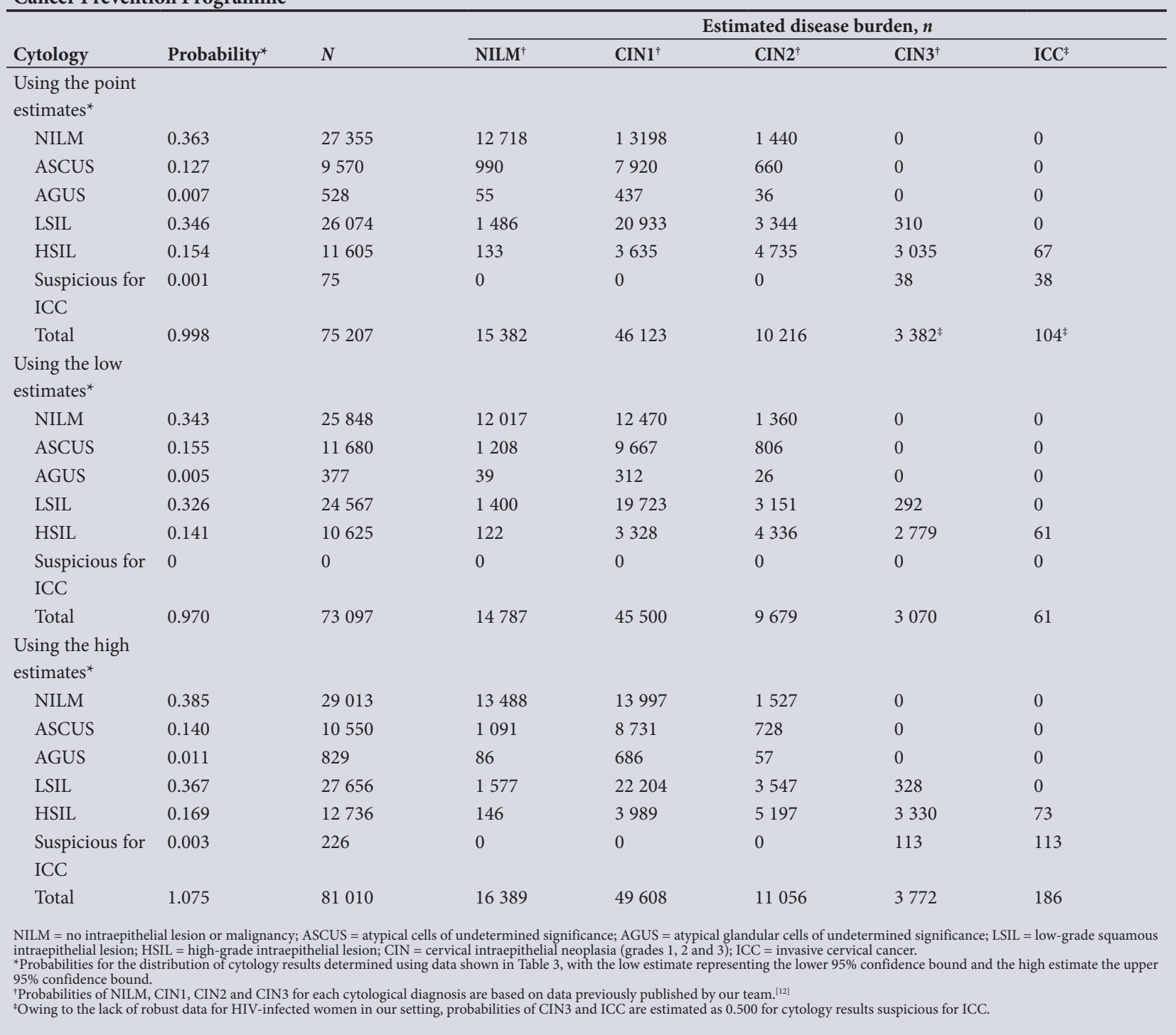

screening programme was established, the annual number of Pap smears performed across the supported sites has increased by $\sim 80 \%$ from 3220 (in eight sites) to 18791 (in 26 sites). We believe that our integrated model, coupled with training and mentorship support, has facilitated this expansion.
Additionally, an integrated model may help to circumvent the 6 - 9-month waiting period for evaluation and treatment of preinvasive disease common in public gynaecology referral centres and may also reduce follow-up losses. Our decision to train medical officers (rather than specialist gynaecologists) has proved useful, 
particularly at lower-level health facilities, where women can now undergo cervical colposcopy (evaluating abnormal cytology results through magnification of the cervix and guided biopsy) and treatment for preinvasive cervical disease. Mobile units (see 'Methods') have also extended a broad range of reproductive health services to under-served areas and are an integral component of our programme.

Despite the rapid expansion of our service delivery model, overall access to cervical screening and prevention and treatment of ICC in SA remains suboptimal. We also acknowledge the challenges of integrated service delivery models, including numerous ways in which integration may stretch the capacity of individual clinicians. Additionally, our service delivery approach would not be relevant for HIV-uninfected women, for whom different strategies may be required (e.g. integration of cervical screening services within antenatal and/or postnatal care). Furthermore, we note several limitations of our model-based analysis. First, our model is based on data obtained primarily from urban and periurban clinical sites, which may not be fully representative of rural settings. Second, although we have provided low and high estimates (based on 95\% CIs) of the burden of disease to account for the uncertainty in our point estimates, the number of cases of ICC in our analysis dataset was low, leading to wide confidence bands and large ranges in the model estimates for ICC. Finally, our model-based analysis does not account for attrition from care, and we have not estimated the cost or cost-effectiveness of the integrated approach.

\section{Conclusion}

We estimate that among $75000 \mathrm{HIV}$-infected women, there were 46123 cases of CIN1 and 13702 cases of CIN2+, confirming a high burden of cervical disease in this population, where nearly one in five women was thought to have high-grade, preinvasive disease and more than 100 cases of ICC were estimated. We also describe an integrated service delivery model that, if expanded, may provide greater and more equitable access to cervical cancer prevention for HIV-infected women in SA. Cervical cancer incidence is projected to rise in LMICs in the coming decade, particularly in countries with a high burden of HIV. Public sector programmes that rapidly increase screening coverage, strengthen linkages between screening and treatment, and improve treatment outcomes for this preventable cancer are urgently needed.

Acknowledgements. The authors acknowledge with thanks the strategic vision and funding support provided by Right to Care's leadership. In particular, we thank Dr Ian Sanne for his support of the programme. We also acknowledge the SA National Health Laboratory Service and the DoH. Portions of the data used in this article were presented at the 7th SA AIDS Conference in June 2015 and published as abstract 212.
Author contributions. CJC conceived and designed the study and drafted the manuscript. BG, SL, MM, MF, SB, AS, SR, SW, NR and PM participated in data collection and provided critical input on the manuscript. CF conceived the study, participated in the design and data collection, and provided critical input on the manuscript. All authors approved the final version of the manuscript for publication.

Funding. Funding support for operational and research activities was provided by USAID PEPFAR (grant nos 674-A-00-08-00007 and 674-A12-00020), USAID PHE (grant no. PHE ZA.09.0265), the US National Institutes of Health (grant no. 5D43CA153715-024), and the First for Women Insurance Company.

Conflicts of interest. None.

1. Ginsberg GM, Lauer JA, Zelle S, Baeten S, Baltussen R. Cost effectiveness of strategies to combat breast, cervical, and colorectal cancer in sub-Saharan Africa and South East Asia: Mathematical modelling study. BMJ 2012;344:e614. https://doi.org/10.1136/bmj.e614

2. Bray F, Ren JS, Masuyer E, Ferlay J. Global estimates of cancer prevalence for 27 sites in the adult population in 2008. Int J Cancer 2013;132(5):1133-1145. https://doi.org/10.1002/ijc.27711

3. Joint United Nations Programme on HIV/AIDS (UNAIDS). Global Factsheets 2015. http://aidsinfo. unaids.org/ (accessed 8 May 2017)

4. Denny LA, Franceschi S, de Sanjose S, Heard I, Moscicki AB, Palefsky J. Human papillomavirus, human immunodeficiency virus and immunosuppression. Vaccine 2012;30(Suppl 5):F168-F174. https://doi.org/10.1016/..vaccine.2012.06.045

5. Simonds HM, Wright JD, du Toit N, Neugut AI, Jacobson JS. Completion of and early response to chemoradiation among human immunodeficiency virus (HIV)-positive and HIV-negative patients with locally advanced cervical carcinoma in South Africa. Cancer 2012;118(11):2971-2979. https:// with locally advanced cervicalc
doi.org/10.1002/cncr.26639

6. Massad LS, Fazzari MJ, Anastos K, et al. Outcomes after treatment of cervical intraepithelial neoplasia Massad LS, Fazzari M), Anastos K, et al. Outcomes after treatment of cervical intraepithelial neoplasia
among women with HIV. J Low Genit Tract Dis 2007;11(2):90-97. https://doi.org/10.1097/01.

lgt.0000245038.06977.a7
7. Murray CJ, Ortblad KF, Guinovart C, et al. Global, regional, and national incidence and mortality for HIV, tuberculosis, and malaria during 1990 - 2013: A systematic analysis for the Global Burden of Disease Study 2013. Lancet 2014;384(9947):1005-1070. https://doi.org/10.1016/S0140-6736(14)60844-8

8. Ervik MLF, Ferlay J, Mery L, Soerjomataram I, Bray F. Cancer Today. Lyon: International Agency for Research on Cancer, 2016. http://gco.iarc.fr/today (accessed 24 November 2016).

9. Goldie SJ, Gaffikin L, Goldhaber-Fiebert JD, et al. Cost-effectiveness of cervical-cancer screening in five developing countries. N Engl J Med 2005;353(20):2158-2168. https://doi.org/10.1056/NEJMsa044278

0. Gakidou E, Nordhagen S, Obermeyer Z. Coverage of cervical cancer screening in 57 countries: Low average levels and large inequalities. PLoS Med 2008:5(6):e132. https://doi.org/10.1371/journal. pmed.0050132

11. Wake RM, Rebe K, Burch VC. Patient perception of cervical screening among women living with human immuno-deficiency virus infection attending an antiretroviral therapy clinic in urban South human immuno-deficiency virus infection attending an antiretroviral therapy clinic in
Africa. J Obstet Gynaecol 2009;29(1):44-48. https://doi.org/10.1080/01443610802484070

12. Firnhaber C, Mayisela N, Mao L, et al. Validation of cervical cancer screening methods in HIV positive wirnhen from Johannesburg South Africa. PLoS One 2013;8(1):e53494. https://doi.org/10.1371/
women

13. Apgar BS, Zoschnick L, Wright TC Jr. The 2001 Bethesda System terminology. Am Fam Physician Apgar BS, Zoschnick L,
2003;68(10):1992-1998.

14. Biggar RJ, Chaturvedi AK, Goedert JJ, Engels EA for the HIV/AIDS Cancer Match Study. AIDS-related cancer and severity of immunosuppression in persons with AIDS. J Natl Cancer Inst 2007;99(12):962 972. https://doi.org/10.1093/jnci/djm010

15. Franceschi S, Jaffe H. Cervical cancer screening of women living with HIV infection: A must in the era of antiretroviral therapy. Clin Infect Dis 2007;45(4):510-513. https://doi.org/10.1086/520022

6. Brower V. AIDS-related cancers increase in Africa. J Natl Cancer Inst 2011;103(12):918-919. https:// doi.org/10.1093/jnci/djr235

7. Casper C. The increasing burden of HIV-associated malignancies in resource-limited regions. Annu Rev Med 2011;62:157-170. https://doi.org/10.1146/annurev-med-050409-103711

18. Abraham AG, D'Souza G, Jing Y, et al. Invasive cervical cancer risk among HIV-infected women: Abraham AG, D'Souza G, Jing Y, et al. Invasive cervical cancer risk among HIV-infected women: 2013;62(4):405-413. https://doi.org/10.1097/QAI.0b013e31828177d7

19. Chirenje ZM. HIV and cancer of the cervix. Best Pract Res Clin Obstet Gynaecol 2005;19(2):269-276. https://doi.org/10.1016/j.bpobgyn.2004.10.002

20. Einstein MH, Phaeton R. Issues in cervical cancer incidence and treatment in HIV. Curr Opin Oncol 2010;22(5):449-455. https://doi.org/10.1097/QAI.0000000000000685

Accepted 23 October 2017. 